\title{
Syphilis Infection During Pregnancy will Increases the Risk of Hypertensive Disorders of Pregnancy- Results from A Case-Control Study in China
}

\section{Xiangyi Zhang}

Jilin University School of Public Health

Huixin Yang

Jilin University School of Public Health

Lixin Sun

Shenzhen Sami International Medical Center

Jing Hu

Jilin University School of Public Health

Mujinyan Li

Jilin University School of Public Health

Fande Li

Jilin University School of Public Health

Shuqi An

Jilin University School of Public Health

Siwen Zhang

Jilin University School of Public Health

Taijun Wang

Jilin University School of Public Health

Qing Zhen ( $\square$ zhenqing@jlu.edu.cn )

Jilin university School of Public Health https://orcid.org/0000-0002-2816-4539

Research article

Keywords: syphilis, HDP, pregnancy, age, anemia

Posted Date: January 18th, 2021

DOI: https://doi.org/10.21203/rs.3.rs-146932/v1

License: (1) (1) This work is licensed under a Creative Commons Attribution 4.0 International License.

Read Full License 


\section{Abstract}

\section{Background}

The incidence of pregnancy syphilis remains at a high level in China. Studies investigating the influencing factors of pregnancy syphilis are mainly concentrated in economically developed areas. Limited studies were conducted in northern China. This study mainly aimed to explore the related factors of pregnancy syphilis and examine the association with the risk of Hypertensive disorders of pregnancy.

\section{Methods}

This case-control study compared pregnancy syphilis cases, matched (1:1) to non-syphilitic pregnant women controls by age, which was diagnosed in Changchun between January 2014 and December 2018. Multivariable logistic regression was used to identify potential correlates of HDP in syphilis populations. Then a propensity score-matched method was conducted to match pregnancy syphilis with and without HDP, and further we explore the related factors of HDP in syphilis by multivariable logistic regression.

\section{Results}

In our study, the median age of syphilis in the case group was 28 years (range 16-53 years). A high rate of syphilis-HDP co-infection $\varangle 49 / 349,12.3 \% \bigotimes$ was observed in syphilis patients and further research revealed an association between syphilis and HDP. Multivariate analysis indicated HDP in syphilis was independently associated with Dysmenorrhea [adjusted odds ratio (a OR) 5.413, 95\% confidence interval (Cl) 2.410-12.158], Maternal anemia (a OR 3.808, 95\% Cl 1.833-7.911), Total cholesterol (a OR 3.144, 95\% Cl 1.485-6.655).

\section{Conclusion}

Syphilis infection in pregnancy associate with the increased risk of hypertensive disorders of pregnancy, attention should also be paid to the occurrence of HDP in the lower age group, to further prevent the occurrence of stroke, cardiovascular disease. Besides, Dysmenorrhea, Maternal anemia, Total cholesterol may be risk factors for HDP in syphilis.

\section{Background}

According to estimates by the World Health Organization (WHO) in 2007, pregnancy syphilis remains a public health problem worldwide and proposed to eliminate mother-to-child transmission of syphilis by 2030(1). Globally, approximately two million pregnant women are infected with syphilis each year and nearly $90 \%$ of the cases occur in developing countries, less than $10 \%$ have not been diagnosed and treated (2-4). Syphilis left untreated, or inadequately treated, transmission of syphilis from mother-tochild during pregnancy can result in serious adverse outcomes for the unborn child in up to $80 \%$ of cases, such as congenital syphilis (CS), which can cause severe sequelae or fetal, neonatal, or infant death $(5,6)$. Relevant researches were reported that different provinces in China suggest progress toward the 
elimination of Mother-to-child transmission (MTCT) of syphilis, the incidence of pregnancy syphilis remains at a high level $(7,8)$. Previous research on pregnancy syphilis in China mainly focused on economically developed areas, such as Shenzhen and Shanghai, few studies were conducted in northern China $(9,10)$. Therefore, it is necessary to discuss the influencing factors of pregnancy syphilis in northern China.

On the other hand, previous large studies suggested that co-infection with syphilis would increase the risk of hepatitis $\mathrm{C}$ virus (HCV), hepatitis B virus (HBV), and human immunodeficiency virus (HIV) infection(11-13). However, in the related studies of the factors associated with syphilis in pregnancy, a study was reported an increased incidence of hypertension in the syphilis group(14). 25-45\% of women with an HDP will develop hypertension within five years of delivery in the normal population(15). Corresponding in pregnancy syphilis, whether syphilis infection is related to the risk of HDP remains unclear. Therefore, we are concerned about the occurrence of HDP in pregnant syphilis.

HDP and syphilis are inflammatory conditions can contribute significantly to adverse maternal and fetal outcomes $(3,16)$, women with HDP have increased risks of post-pregnancy hypertension, Cardiovascular Disease (CVD), stroke, and the long-term risk of cardiovascular disease for children $(17,18)$. And HDP is a major cause of maternal, fetal, neonatal morbidity and mortality, which became the second leading cause of maternal death in China(19). Although significant efforts have been made, a study in Ukraine in 2019 found hypertensive disorders of pregnant women were 2.4 times more common in women of the main group, while a greater percentage of this disorder was recorded among women in the main group who were ill with syphilis (35\%)(20). This reminds us, syphilis with HDP may lead to more serious cardiovascular disease. At present, researches about other factors associated with syphilis infection, such as Chronic disease-related indicators, which potentially affect the CVD, are limited.

The main objectives of this study were to explore the associated factors of syphilis in pregnant women, examine the association with the risk of Hypertensive disorders of pregnancy and understand the related factors of HDP in pregnancy syphilis. Providing advice and measures to prevent the occurrence of HDP in syphilis women during pregnancy and diseases such as CVD, stroke after pregnancy to promote the health of future generations.

\section{Methods}

\section{Study population and data collection}

A retrospective case-control study was conducted in Changchun Maternity Hospital, Jilin Province, China, from January 2014 to December 2018. During this period, a total of 109609 inpatients were screened for syphilis infection by serological tests. All of them, 412 had positive serological tests for syphilis and ultimately were diagnosed with syphilis based on clinical presentation, clinical data, and medical history. Excluding 8 cases with incomplete or missing information and 6 cases with uncertain serological examination results, in total 398 patients were included in the case group. Of the remaining 109,197 nonsyphilis inpatients in the hospital, 398 inpatients were matched (1:1) by age were randomly selected to be 
included in the control group (Fig. 1). Then according to the 2013 ACOG guideline HDP diagnostic criteria, a total of 49 cases diagnosed as HDP in the case group were included in the HDP group, 147 of the remaining 349 syphilis cases without HDP were selected to be included in the non-HDP group using the propensity score method after adjusting the covariates, and subsequent analysis of HDP related factors were performed. The research design has been approved by the Institutional Ethics Committee of Changchun City Maternity Hospital, Jilin Province, and was found to comply with national legislation and the Declaration of Helsinki guideline.

\section{TPPA: Treponema pallidum particle agglutination test}

\section{RPR: Rapid plasma responsive ring card test}

\section{Variable Definition}

In this study, we analyzed some variables related to pregnancy syphilis and HDP in the syphilis case group. All blood samples were collected early in pregnancy, and related blood biochemical indicators were tested. Define changes in relevant blood biochemical characteristics according to their respective diagnostic criteria, such as C-reactive protein (CRP), RPR titer, Total cholesterol (TC). Dysmenorrhea was defined as acute menstrual pain around or during menstruation, often accompanied by other symptoms, such as gastrointestinal dysfunction, irritability, depression, etc; Gestational diabetes was defined as a glucose intolerance that develops during pregnancy; Viral hepatitis was defined as a disease caused by a variety of hepatitis viruses infected by liver cells and mainly manifests as nausea, pain in the liver area, loss of appetite, and fatigue; Maternal anemia was defined as Hemoglobin level $<11.0 \mathrm{~g} / \mathrm{dL}$ during pregnancy; Abortion was defined as spontaneous abortion during pregnancy; Adverse pregnancy outcomes (APOs) were defined as early fetal deaths, stillbirths, neonatal death, preterm birth, low birth weight, and congenital syphilis (CS); Premature rupture of membranes (PROM) was defined as when fetal chorio-amnionic membranes rupture at any time before the onset of true labor; Turbid amniotic fluid was defined as meconium, fetal fat, cholestasis, mixed with amniotic fluid, including bloody amniotic fluid; Umbilical cord abnormality was defined as fetal blood flow is reduced or interrupted due to altered structure or function of the umbilical cord; Un-treatment in pregnancy was defined as not receiving or insufficient treatment; Premature abruption was defined as the premature separation of the placenta before birth after 20 weeks of gestation.

\section{Clinical diagnosis of syphilis and HDP}

This study clinically diagnosed syphilis by combining serological diagnosis and disease history (including clinical features and/or patient history), which is consistent with our previous studies $(21,22)$. Laboratory tests for syphilis were performed using the Treponema pallidum particle agglutination test (TPPA) and the rapid plasma responsiveness test (RPR) to diagnose patients with syphilis(23): (1)A history of syphilis infection $₫ 2 \bigotimes$ Clinical signs and symptoms of each stage of syphilis $₫ 3 \otimes T P P A$ positive and RPR positive and simultaneously perform RPR titer determination. 
Hypertensive disorder of pregnancy, which is defined as hypertension diagnosed after 20 weeks of pregnancy, including: pregnancy-induced hypertension syndrome; pre-eclampsia; eclampsia. Other obstetric complications: uterine rupture, placenta previa, etc, medical complications (heart disease, embolism, etc.), kidney disease (urinary tract infection and chronic kidney disease), lung disease (Upper respiratory tract infection), diabetes (including gestational diabetes), AIDS, cancer, etc were excluded (24).

\section{Propensity score matching analysis}

Propensity score (PS) refers to the possibility that under certain covariate conditions, an observation object may receive a certain treatment (or exposure) factor. Propensity score matching is to find one or more individuals with the same or very close PS value from the control group for each individual in the case group as a control, finally the confounding variables of the two groups tend to be balanced and comparable. Due to some variables in the HDP and the non-HDP group are not completely proportionate, PSM is used to match the HDP patients in the syphilis case group with non-HDP patients to reduce the potential confounding bias. Before matching, the $\chi 2$ test was used to test the balance of potential confounding factors in the two groups to include variables for matching, the R package "Matchlt" was used to conduct propensity score matching analysis. Finally, 49 HDP patients and 147 non-HDP patients were included for follow-up analysis.

\section{Statistical analysis}

All statistical analyses were performed using IBM SPSS version 24.0 for windows and R Studio version 4.0.3. Chi-square tests were used to compare the distributions of Sociodemographic and clinical characteristics between case and control group. Univariate and multivariate logistic regression analyses were performed, calculated adjusted odds ratios, and $95 \%$ confidence intervals. A Multivariate logistic regression model was used to identify potential correlates of HDP in syphilis populations, analyze the associated factors of HDP after propensity score matching by adjusting for the confounders. A p-value of $\leq 0.05$ was taken as statistically significant.

\section{Results}

\section{Sociodemographic and clinical characteristics}

The distributions of socio-demographic and clinical characteristics of pregnancy syphilis cases and controls are shown in Table 1, and there were no significant differences between cases and controls on the matched variable (Age). Compared to the control group, the median age of pregnancy syphilis was 28 years (range, $16-53$ years). Most cases and controls are in the $25-29$ age group (39.2\%;41.0\%), which is consistent with the results of a 10-year review in Shenzhen, China(25).

There were significant differences in the distribution of study places, the urban population in the case group accounted for $62.3 \%$ (248/398). Between the two groups, there are also significant differences in the lack of paid occupations, the great majority of which are lack of paid occupations $(71.6 \% ; 62.6 \%)$. 
Compared to the non-syphilitic pregnant women control group, Abortion, Turbid amniotic fluid, APOs have significant differences $(P<0.01)$. Among the biochemical indicators, the contents of CRP are different in the two groups $(p<0.05)$. Prevalence of HDP $(n=49,12.3 \%)$ and viral hepatitis $(n=22,3.8 \%)$ increased significantly in the case group, while the prevalence of $\operatorname{HDP}(n=15,3.8 \%)$ and viral hepatitis $(n=4,1.0 \%)$ was lower in the control group $(p<0.05)$

Table 1. The distributions of General demographic characteristics of pregnancy syphilis cases and controls.

\begin{tabular}{|c|c|c|c|}
\hline & $\mathrm{N}(\%)$ & & \\
\hline \multirow[t]{2}{*}{ Characteristic } & Case & Control & $P$ value \\
\hline & $\mathrm{N}=398(\%)$ & $\mathrm{N}=398(\%)$ & \\
\hline \multicolumn{4}{|l|}{ Age, years } \\
\hline (mean \pm Standard deviation) & $28.40 \pm 5.357$ & $28.97 \pm 4.861$ & - \\
\hline \multicolumn{4}{|l|}{ Age, years } \\
\hline $15-24$ & $94(23.6)$ & $73 \otimes 18.3 \rrbracket$ & 0.207 \\
\hline $25-29$ & $156(39.2)$ & $163 \rrbracket 41.0 \rrbracket$ & \\
\hline $30-34$ & $91 \rrbracket 22.9 \rrbracket$ & $109 \llbracket 27.4 \rrbracket$ & \\
\hline $35-53$ & 57囚14.3囚 & 53囚13.3囚 & \\
\hline
\end{tabular}

Table1. Continued 


\begin{tabular}{|c|c|c|c|}
\hline & $\mathrm{N}(\%)$ & & \\
\hline \multirow[t]{2}{*}{ Characteristic } & Case & Control & $P$ value \\
\hline & $\mathrm{N}=398(\%)$ & $\mathrm{N}=398(\%)$ & \\
\hline Absence of paid occupation & 285ه71.6》 & & \\
\hline Yes & $113 \llbracket 28.4 \rrbracket$ & $249 \otimes 62.6 \rrbracket$ & 0.007 \\
\hline No & & $149 \rrbracket 37.4 \rrbracket$ & \\
\hline Present residence & $248 \bowtie 62.3 \rrbracket$ & $279 \otimes 70.1 \rrbracket$ & 0.020 \\
\hline $\begin{array}{l}\text { City } \\
\text { rural }\end{array}$ & 150®37.7凶 & 119ه29.9凶 & \\
\hline \multicolumn{4}{|l|}{ Dysmenorrhea } \\
\hline Yes & $79 \rrbracket 19.8 \rrbracket$ & $94 \llbracket 23.7 \rrbracket$ & 0.198 \\
\hline No & $319 \llbracket 80.2 \rrbracket$ & $303 \rrbracket 76.3 \rrbracket$ & \\
\hline Abortion & & & $\square 0.01$ \\
\hline 0 & $186 \rrbracket 46.7 \rrbracket$ & $259 \llbracket 65.1 \rrbracket$ & \\
\hline 1 & $120 \rrbracket 24.4 \rrbracket$ & $97 \varangle 30.2 \rrbracket$ & \\
\hline$\geq 2$ & $96 \rrbracket 28.9 \rrbracket$ & $42 \varangle 4.7 \rrbracket$ & \\
\hline HDP & & & $\square 0.01$ \\
\hline Yes & $49 \rrbracket 12.3 \rrbracket$ & $15 \otimes 3.8 \rrbracket$ & \\
\hline No & $349 \llbracket 87.7 \rrbracket$ & $383 \otimes 96.2 \bigotimes$ & \\
\hline Maternal anemia & & & 0.821 \\
\hline Yes & $129 \llbracket 32.4 \rrbracket$ & $132 \varangle 33.2 \rrbracket$ & \\
\hline No & $269 \rrbracket 67.6 \rrbracket$ & $266 \rrbracket 66.8 \rrbracket$ & \\
\hline \multicolumn{4}{|l|}{ Gestational diabetes } \\
\hline Yes & $41 \otimes 10.3 \rrbracket$ & $49 \rrbracket 12.3 \rrbracket$ & 0.371 \\
\hline No & $357 \llbracket 89.7 \rrbracket$ & $349 \llbracket 87.7 \rrbracket$ & \\
\hline \multicolumn{4}{|l|}{ Viral hepatitis } \\
\hline Yes & $22 \otimes 5.5 \rrbracket$ & $4 \rrbracket 1.0 \rrbracket$ & $0.001 *$ \\
\hline No & $376 \rrbracket 94.5 \rrbracket$ & $394 \varangle 99.0 \rrbracket$ & \\
\hline \multicolumn{4}{|l|}{ PROM } \\
\hline Yes & $\begin{array}{l}71 \rrbracket 17.8 \rrbracket \\
\text { Page } 7 / 20\end{array}$ & $69 \otimes 17.3 \rrbracket$ & 0.852 \\
\hline
\end{tabular}




\begin{tabular}{|c|c|c|c|}
\hline No & $327 \rrbracket 82.2 \rrbracket$ & $329 \bowtie 82.7 \rrbracket$ & \\
\hline \multicolumn{4}{|l|}{ Placental abruption } \\
\hline Yes & $2(0.5)$ & $3(0.8)$ & $1.000 *$ \\
\hline No & 396 (99.5) & $396(99.5)$ & \\
\hline \multicolumn{4}{|l|}{ Table1. Continued } \\
\hline \multicolumn{4}{|c|}{$\mathrm{N}(\%)$} \\
\hline \multirow[t]{2}{*}{ Characteristic } & Case & Control & $P$ value \\
\hline & $\mathrm{N}=398(\%)$ & $\mathrm{N}=398(\%)$ & \\
\hline \multicolumn{4}{|l|}{ Turbid amniotic fluid } \\
\hline High & $109 \llbracket 27.4 \rrbracket$ & $28 \otimes 7.0 \otimes$ & 00.01 \\
\hline Low & $289 \bigotimes 72.6 \rrbracket$ & $370 \varangle 93.0 \rrbracket$ & \\
\hline \multicolumn{4}{|c|}{ Umbilical cord abnormality } \\
\hline Yes & $99 \rrbracket 24.9 \rrbracket$ & $115 \varangle 28.9 \rrbracket$ & 0.201 \\
\hline No & $299 \bigotimes 75.1 \rrbracket$ & $283 \otimes 71.1 \rrbracket$ & \\
\hline \multicolumn{4}{|l|}{ APOs } \\
\hline Yes & $52(13.1)$ & $3(0.8)$ & {$\left[0.01^{*}\right.$} \\
\hline No & $346(86.9)$ & $395(99.2)$ & \\
\hline \multicolumn{4}{|l|}{ CRP } \\
\hline Normal & $276 \bowtie 69.3 \rrbracket$ & $332 \llbracket 83.4 \rrbracket$ & 00.01 \\
\hline Elevated (>10mg/L) & $122 \llbracket 30.7 \rrbracket$ & $66 \otimes 16.6 \rrbracket$ & \\
\hline
\end{tabular}

* Corrected chi-square value

APOs, Adverse pregnancy outcome; CRP, High-sensitivity C-reactive protein;

PROM, Premature rupture of membranes; HDP, Hypertensive disorders of pregnancy;

\section{Logistic Regression Modelling}

In the Univariate analysis, all factors related to syphilis were included in the Multivariable model (based on $P$ value $\leq 0.05$; Table 2). After adjustment age, Absence of paid occupation, Non-urban population, variables that were retained in the final multivariable model (Table 2) were: Absence of paid occupation 
(OR 1.536, Cl 1.106-2.134) $\square$ Abortion 1 time and $\geq 2$ times (OR 1.770, Cl 1.242-2.523)(OR 3.128, Cl 2.015-4.857) \HDP (OR 2.017, Cl 1.042-3.906)【Viral hepatitis (OR 4.702, Cl 1.544-14.318)םTurbid amniotic fluid (OR 2.945, Cl 1.762-4.923) $\square$ APOs (OR 7.428, Cl 2.137-25.820), CRP ( $\geq 10 \mathrm{mg} / \mathrm{L})$ (OR $1.817, \mathrm{Cl} 1.249-2.643)$.

Table 2. Logistic regression model results of pregnancy syphilis cases and controls.

\begin{tabular}{|c|c|c|c|c|c|c|}
\hline \multirow[t]{2}{*}{ Variable } & \multicolumn{2}{|c|}{ Bivariable analysis } & \multirow{2}{*}{$\begin{array}{l}P \\
\text { value }\end{array}$} & \multicolumn{2}{|c|}{ Multivariable analysis } & \multirow{2}{*}{$\begin{array}{l}P \\
\text { value }\end{array}$} \\
\hline & OR & $95 \% \mathrm{Cl}$ & & a OR & $95 \% \mathrm{Cl}$ & \\
\hline Absence of paid occupation & 1.509 & $0.492-0.892$ & 0.007 & 1.536 & $1.106-2.134$ & 0.010 \\
\hline Non-urban population & 1.418 & $1.056-1.905$ & 0.02 & - & - & - \\
\hline \multicolumn{7}{|l|}{ history of abortion } \\
\hline 0 & ref & - & - & - & - & - \\
\hline 1 & 1.723 & $1.242-2.390$ & 0.001 & 1.770 & $1.242-2.523$ & 0.002 \\
\hline$>=2$ & 3.050 & 2.023-4.599 & 0.013 & 3.128 & $2.015-4.857$ & $<0.001$ \\
\hline HDP & 3.585 & $1.957-6.508$ & $<0.001$ & 2.017 & $1.042-3.906$ & 0.037 \\
\hline Viral hepatitis & 5.763 & $1.968-16.881$ & 0.001 & 4.702 & $1.544-14.318$ & 0.006 \\
\hline Turbid amniotic fluid & 4.984 & $3.201-7.761$ & $<0.001$ & 2.945 & $1.762-4.923$ & $<0.001$ \\
\hline APOs & 19.788 & $6.125-63.928$ & $<0.001$ & 7.428 & $2.137-25.820$ & 0.002 \\
\hline $\mathrm{CRP}(\geq 10 \mathrm{mg} / \mathrm{L})$ & 2.224 & $1.584-3.122$ & $<0.001$ & 1.817 & $1.249-2.643$ & 0.002 \\
\hline
\end{tabular}

a OR, Adjusted odds ratio; $\mathrm{Cl}$, confidence interval; HDP, Hypertensive disorders of pregnancy; APOs, Adverse pregnancy outcome; CRP, High-sensitivity C-reactive protein;

\section{Analysis of related factors of HDP in syphilis}

The results of multivariate analysis of syphilis-related factors demonstrated that patients with syphilis had an increased risk of HDP (aOR2.017,95\% Cl1.042-3.906), so we further explored the factors related to HDP. Using the propensity score method, after controlling for age, occupation, ethnicity, parity, and urine protein, 49 HDP patients were matched 1:3 with non-HDP patients, and a total of 147 non-HDP patients were matched. Univariate and Multivariate analyses were performed on 49 HDP patients and 147 nonHDP patients (Table 3).

The mean age of the HDP group (27.76) was lower than that of the Non-HDP group (28.13), and there was no significant difference in the incidence of the age groups $(P<0.05)$. In pregnant syphilis patients, 
compared with the Non-HDP group, Dysmenorrhea (42.9\%), Maternal anemia (61.2\%), Total cholesterol (55.1\%) of the HDP group were higher than the Non-HDP group: Dysmenorrhea (13.6\%), Maternal anemia (29.3\%), Total cholesterol (34.0\%).

Dysmenorrhea history remained positively associated with HDP [adjusted odds ratio [(a OR) 5.413, 95\%

$\mathrm{Cl}$ 2.410-12.158]. Associations between HDP and Maternal anemia were noted (a OR)3.808, 95\% Cl1.8337.911). Compared with Normal Total cholesterol, Total cholesterol ( $>=5.69 \mathrm{mmol} / \mathrm{L})$ was positively correlated with HDP [(a OR) 3.144, 95\% Cl 1.485-6.655].

Table 3. Univariate and multivariate analysis results of HDP cases and control. 


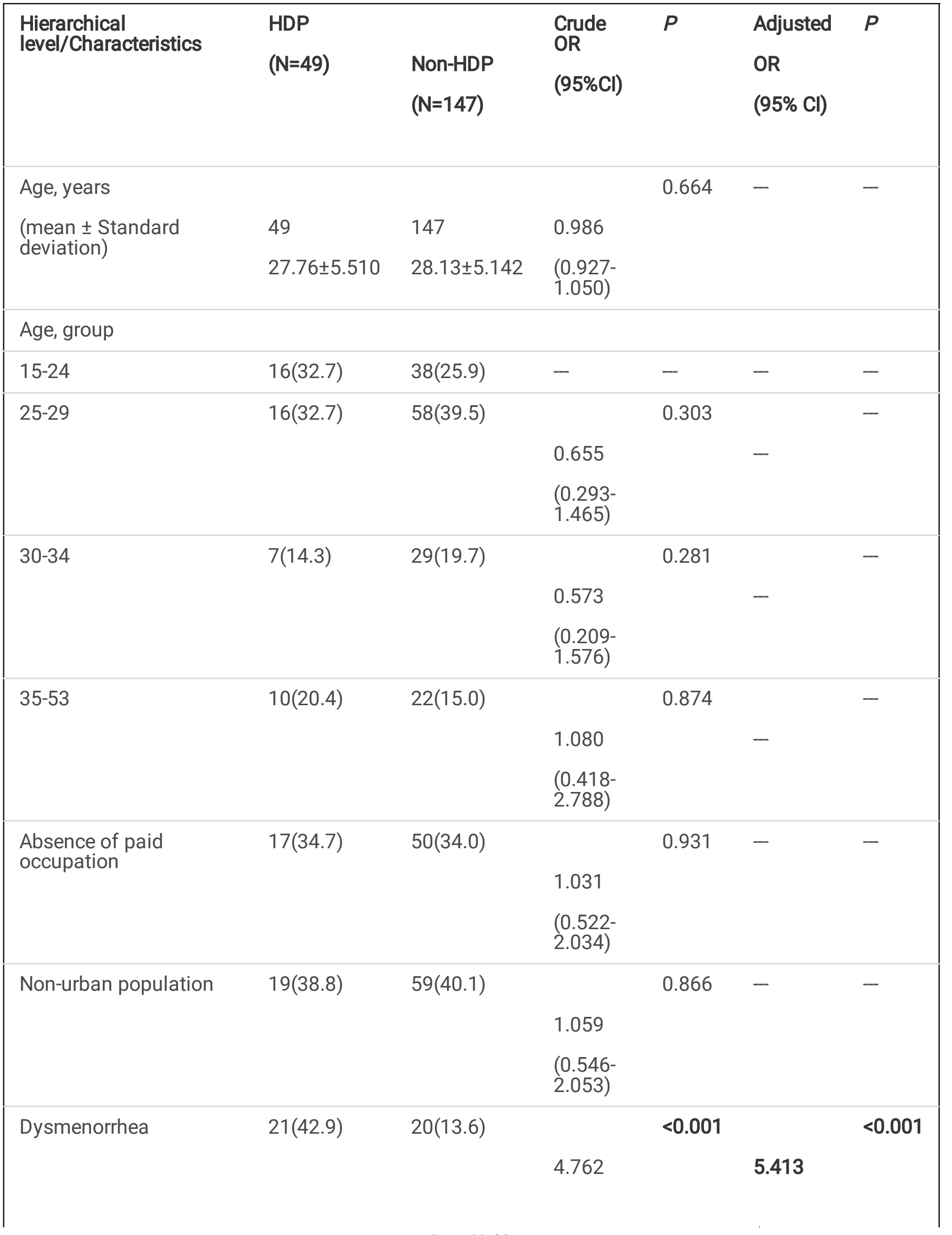




\begin{tabular}{|c|c|c|c|c|c|c|}
\hline & & & $\begin{array}{l}(2.280- \\
9.947)\end{array}$ & & \multicolumn{2}{|c|}{$\begin{array}{l}(2.410- \\
12.158)\end{array}$} \\
\hline \multicolumn{7}{|l|}{ Abortion } \\
\hline 0 & $20(40.8)$ & $75(51.0)$ & - & - & - & -- \\
\hline 1 & $17(34.7)$ & 41(27.9) & $\begin{array}{l}1.555 \\
(0.734- \\
3.293)\end{array}$ & 0.249 & - & -- \\
\hline$\geq 2$ & $12(24.5)$ & $31(21.1)$ & $\begin{array}{l}1.452 \\
(0.634- \\
3.326)\end{array}$ & 0.378 & - & - \\
\hline $\begin{array}{l}\text { Un-treatment in } \\
\text { pregnancy }\end{array}$ & $37(75.5)$ & 118(80.3) & $\begin{array}{l}1.320 \\
(0.613- \\
2.843)\end{array}$ & 0.479 & - & -- \\
\hline
\end{tabular}

Table3. Continued 


\begin{tabular}{|c|c|c|c|c|c|c|}
\hline $\begin{array}{l}\text { Hierarchical } \\
\text { level/Characteristics }\end{array}$ & $\begin{array}{l}\text { HDP } \\
(\mathrm{N}=49)\end{array}$ & $\begin{array}{l}\text { Normal } \\
(\mathrm{N}=349)\end{array}$ & $\begin{array}{l}\text { Crude OR } \\
(95 \% \mathrm{Cl})\end{array}$ & $P$ & $\begin{array}{l}\text { Adjusted } \\
\text { OR } \\
(95 \% \mathrm{Cl})\end{array}$ & $P$ \\
\hline RPR titer $₫ \geq 1: 8 \rrbracket$ & $22(44.9)$ & $45(30.6)$ & $\begin{array}{l}1.847 \\
(0.951-3.585)\end{array}$ & 0.070 & - & - \\
\hline APOs & $9 \rrbracket 18.4 \rrbracket$ & 19(12.9) & $\begin{array}{l}1.516 \\
(0.636-3.614)\end{array}$ & 0.348 & - & - \\
\hline Placental abruption & $1(2.0)$ & $1(0.7)$ & $\begin{array}{l}3.042 \\
(0.187,49.567)\end{array}$ & 0.435 & - & - \\
\hline Gestational diabetes & $2(4.1)$ & $15(10.2)$ & $\begin{array}{l}0.374 \\
(0.083,1.699)\end{array}$ & 0.203 & - & - \\
\hline Maternal anemia & $30(61.2)$ & $43(29.3)$ & $\begin{array}{l}3.819 \\
(1.943-7.505)\end{array}$ & $<0.001$ & $\begin{array}{l}3.808 \\
(1.833- \\
7.911)\end{array}$ & $<0.001$ \\
\hline $\mathrm{CRP}(\geq 10 \mathrm{mg} / \mathrm{L})$ & 18(36.7) & $48(32.7)$ & $\begin{array}{l}1.198 \\
(0.610-2.353)\end{array}$ & 0.601 & - & - \\
\hline $\begin{array}{l}\text { Total cholesterol } \\
(>5.69 \mathrm{mmol} / \mathrm{L})\end{array}$ & $27(55.1)$ & $50(34.0)$ & $\begin{array}{l}2.381 \\
(1.233-4.599)\end{array}$ & 0.010 & $\begin{array}{l}3.144 \\
(1.485- \\
6.655)\end{array}$ & 0.003 \\
\hline
\end{tabular}

a OR, Adjusted odds ratio; $\mathrm{Cl}$, confidence interval; $\mathrm{CRP}$, High-sensitivity C-reactive protein; RPR, rapid plasma reagin ring card test; PROM, Premature rupture of membranes;

APOs, Adverse pregnancy outcome;

\section{Discussion}

Our study described the demographic and clinical characteristics of syphilis patients in China. And studied factors associated with this infection to explore whether the risk of HDP in syphilis populations would increase, then further explore the related factors of HDP in syphilis population. To our knowledge, no other studies investigating risk factors associated with HDP in syphilis populations. We found that increased risk of HDP in the pregnancy syphilis population and dysmenorrhea, maternal anemia, total cholesterol may be a risk factor for HDP in syphilis. 
Overall, our findings were comparable to the findings of other studies on pregnancy syphilis(26). The demographic characteristics of our study participants corroborated findings of other descriptive analyses on pregnancy syphilis, particularly with the strong 25-34 age group predominance of pregnancy syphilis patients, the majority of which absence of paid occupation(26-28). Although studies have shown that HDP may be associated with the development of inflammatory diseases such as syphilis, many studies have not included the analysis of factors related to the inclusion of HDP in syphilis. In our case-control study, the indicator of HDP in pregnant women was included to examine the association between syphilis and HDP. The results showed that the risk of HDP was increased in patients with syphilis during pregnancy. Therefore, it is very important to further explore the influencing factors of HDP in syphilis populations, which we need to take preventive measures against the occurrence of more serious adverse pregnancy outcomes, stroke and CVD after pregnancy.

Pregnant women with syphilis are mainly from the city, consistent with current findings, Assefa reported a relatively high incidence of this disease in urban pregnant women(29). However, we have not concluded that the area of residence is an independent risk factor for syphilis in pregnancy. Our study found that half of all pregnant syphilis patients have a history of abortion, one-third of the syphilis women have more than 2 abortions, this is consistent with the conclusions obtained from previous studies(30). This reminds us pregnant women with habitual abortion should be forced to carry out syphilis screening before pregnancy. It is well known that pregnancy syphilis can cause adverse pregnancy outcomes in the fetus, like congenital syphilis, and often associated with infectious diseases such as viral hepatitis, our research also proves this conclusion $(3,14)$.

Turbid amniotic fluid is mixed with meconium, blood, pus, or a large amount of tissue exudate so that the color and transparency of amniotic fluid will change significantly, and amniotic fluid will appear turbid. The current case report on congenital syphilis indicates that the maternal turbidity of the amniotic fluid occurs, and the amniocentesis test reveals the syphilis spirochete $(31,32)$. syphilis is an inflammatory disease that causes infection in the uterus or in the amniotic cavity of pregnancy. pregnancy intrauterine infection will eventually spread to the amniotic cavity, causing intra-amniotic cavity infection, a large number of pus cells, white blood cells, and tissue fluid in the amniotic cavity exudate mixed into the amniotic fluid, making the turbid amniotic fluid. Meanwhile, among women with syphilis infection, the concentration of IgM was elevated as was the ratio of lecithin to sphingomyelin in the amniotic fluid. Our findings validate the results of the above study: an increased risk of amniotic fluid turbidity in patients with syphilis during pregnancy.

C-reactive protein (CRP) has long been used as a marker of systemic inflammation as circulating levels of CRP increase several hundred folds within hours of an inflammatory insult(33). What we are paying attention to is that in the case-control study of syphilis in pregnancy, the CRP level in the case group is significantly higher ( $>10 \mathrm{mg} / \mathrm{L})(122 / 398)$ compared with the control group. This is consistent with the conclusions of several related studies $(34,35)$. 
There is a growing body of evidence that hypertension, and vascular disease in general, is an inflammatory disease $(36,37)$. At the same time, studies have shown that there is a certain correlation between hypertension and CRP levels, and the higher the grade of hypertension, the higher the CRP concentration. This also suggests that there is a certain correlation between the occurrence and development of hypertension and the body's inflammatory response(38). Based on this, after we found that the risk of HDP in syphilis patients increased, we explored the related factors and found some results.

A study in China had shown that the incidence of HDP was lowest in 20-29 years old women, and increased obviously in those $>35$ years(39). This is inconsistent with our findings. We found that there was no significant difference in the age distribution of patients with syphilis in syphilis. This indicates that pregnant women of all ages in the syphilis population are at risk of HDP. Therefore, for the view that HDP is more likely to occur in high age groups, we must pay special attention to prevent HDP in low age groups in the prevention of syphilis.

Research also shows the history of dysmenorrhea is a risk factor for HDP in maternal syphilis. Previous researches have shown that the occurrence of hypertensive disorder complicating pregnancy is usually affected by dysmenorrhea or irregular menstruation, which may be due to metabolic syndrome or Vasoactive substances, causing significant effects on blood vessels(40).

In our study, anemia was found to be associated with the development of HDP in people with syphilis. We believe that the cause of anemia in pregnant women may be iron deficiency during pregnancy. Iron deficiency is common in pregnancy. To our knowledge, there is controversy about the relationship between serum iron levels and HDP. Fenzl et al. showed significantly higher serum iron concentrations in 30 women with HDP, compared to 37 healthy pregnant women. The same profile of results was found by Das et al. $(41,42)$. In contrast, two pilot prospective types of research have shown that as the serum iron concentration decreases early in pregnancy, the risk of developing HDP increases $(43,44)$. Therefore, we have reason to believe that the results obtained in this study are supported.

Total cholesterol elevated is related to the risk of HDP in patients with syphilis. To meet the needs of pregnancy, fetal development, childbirth, and postpartum breastfeeding, blood lipid levels during pregnancy will increase by $46-52 \%$ compared to before pregnancy and change accordingly as the number of weeks of pregnancy increases(45). The blood lipid level gradually increased from the $21 \mathrm{st}$ week of gestation and gradually stabilized at a higher level after the 31 st week of gestation. The TC level will increase by about $25 \%$, and the TG level will increase by about 2.5 times compared with healthy women. Excessively elevated TC can lead to adverse pregnancy outcomes. Cuihong Zhao et al. found that TC gradually increased with the aggravation of hypertension during pregnancy and TC is an independent risk factor for normal pregnant women to progress to pregnancy-induced hypertension(46). Disorders of lipid metabolism play an important role in the pathogenesis of pregnancy-induced hypertension by damaging the vascular endothelium. When blood lipid levels increase, lipid peroxidation is enhanced, and excessive oxygen free radicals are formed, which damages vascular endothelial cells. 
The second possible mechanism is that the pathological process of pregnancy-induced hypertension is due to the imbalance of lipoprotein lipase, which leads to abnormal lipid distribution of blood lipids (47). Therefore, we can believe that abnormal blood lipid levels during pregnancy can cause HDP.

\section{Conclusion}

In pregnancy syphilis, the risk of HDP increases. In this study, HDP associated with dysmenorrhea, anemia, total cholesterol elevated. It is vital to comprehensively explore the related factors of pregnancy syphilis, paying special attention to the relationship between syphilis and chronic diseases. We also found that there is no difference in the age distribution of patients with syphilis in patients with HDP, and it is also necessary to prevent and control the occurrence of pregnancy-induced hypertension in young people with syphilis to further prevent the occurrence of diseases, like stroke and CVD.

\section{Declarations}

Ethical Approval and consent to participate: The research design has been approved by the Institutional Ethics Committee of Changchun City Maternity Hospital, Jilin Province (20190109-CC12).

Consent for publication: Not applicable

Availability of data and materials: The datasets used or analyzed during the current study are available from the corresponding author on reasonable request.

Acknowledgments: We appreciated all authors for completing the manuscript.

Authors contributions: YHX, SLX, ZQ conceived the study. HJ, LMJY, LFD, ASQ, ZSW, WTJ involved in data collection and analysis, and all critically reviewed the manuscript. YHX, ZXY, SLX wrote the first draft of the manuscript. All authors reviewed and approved the final version of the manuscript.

Competing interests: The authors have declared that no competing interests exist.

Funding: This research did not receive any specific grant from funding agencies in the public, commercial, or not-for-profit sectors.

\section{References}

1. Meredith S, Hawkes S, Schmid G, Broutet N. The global elimination of congenital syphilis: rationale and strategy for action. Geneva, Switzerland, World Health Organization [WHO], 2007. 2007.

2. Wijesooriya NS, Rochat RW, Kamb ML, Turlapati P, Temmerman M, Broutet N, et al. Global burden of maternal and congenital syphilis in 2008 and 2012: a health systems modelling study. Lancet Global Health. 2016;4(8):e525-e33. 
3. Zhang X, Yu Y, Yang HJ, Xu HY, Vermund SH, Liu KB. Surveillance of Maternal Syphilis in China: Pregnancy Outcomes and Determinants of Congenital Syphilis. Med Sci Monit. 2018;24:7727-35.

4. Arnesen L, Martínez G, Mainero L, Serruya S, Durán P. Gestational syphilis and stillbirth in Latin America and the Caribbean. Int J Gynaecol Obstet. 2015;128(3):241-5.

5. Rowe CR, Newberry DM, Jnah AJ. Congenital Syphilis. A Discussion of Epidemiology, Diagnosis, Management, and Nurses' Role in Early Identification and Treatment. Adv Neonatal Care. 2018;18(6):438-45.

6. Lafond RE, Lukehart SA. Biological basis for syphilis. Clin Microbiol Rev. 2006;19(1):29-49.

7. Zhiyu L, Tingting W, Yuan L, Aihua W, Donghua X, Fanjuan K, et al. Evaluating the progress to eliminate mother-to-child transmission (MTCT) of syphilis in Hunan Province, China: A study based on a health service delivery model. PLOS ONE. 2018;13(9).

8. Chen YF, Ding JP, Yan HJ, Lu J, Ding P, Chen GH, et al. The current status of syphilis prevention and control in Jiangsu province, China: A cross-sectional study. PLoS One. 2017;12(8):e0183409.

9. Cheng JQ, Zhou H, Hong FC, Zhang D, Zhang YJ, Pan P, et al. Syphilis screening and intervention in 500,000 pregnant women in Shenzhen, the People's Republic of China. Sexually Transmitted Infections. 2007;83(5):347.

10. Du L, Li Y, Jin H, Huang C, Gu Y, Zhu L, et al. Prevent Mother-to-Child Transmission (PMTCT) Programs and Enhancement of Maternal Healthcare Infrastructure to Improve Early Detection of Maternal Syphilis in Shanghai, China. Int J Environ Res Public Health. 2019;16(6):1002.

11. Blocker ME, Levine WC, St Louis ME. HIV prevalence in patients with syphilis, United States. Sex Transm Dis. 2000;27(1):53-9.

12. Wandeler G, Gsponer T, Bregenzer A, Gunthard HF, Clerc O, Calmy A, et al. Hepatitis C virus infections in the Swiss HIV Cohort Study: a rapidly evolving epidemic. Clinical infectious diseases: an official publication of the Infectious Diseases Society of America. 2012;55(10):1408-16.

13. McQuillan GM, Townsend TR, Fields HA, Carroll M, Leahy M, Polk BF. Seroepidemiology of hepatitis B virus infection in the United States. 1976 to 1980. Am J Med. 1989;87(3A):5S-10S.

14. Xiao Y, Li SL, Lin HL, Lin ZF, Zhu XZ, Fan JY, et al. Factors associated with syphilis infection: a comprehensive analysis based on a case-control study. Epidemiol Infect. 2016;144(6):1165-74.

15. Veerbeek JHW, Wietske H, Breimer AY, Rijn BB, Van, Koenen SV, Mol BW, et al. Cardiovascular disease risk factors after early-onset preeclampsia, late-onset preeclampsia, and pregnancy-induced hypertension. Hypertension. 2015;65(3):600-6.

16. Organization WH. WHO Recommendations for Prevention and Treatment of Pre-Eclampsia and Eclampsia. Geneva2011.

17. Leanne B, Juan-Pablo $C$, Hingorani AD, Williams DJ. Pre-eclampsia and risk of cardiovascular disease and cancer in later life: systematic review and meta-analysis. Bmj British Medical Journal. 2007;335(7627):974-7. 
18. Brown CM, Pearce SM, Best, Bell, et al. Cardiovascular disease risk in women with pre-eclampsia: systematic;review and meta-analysis. Eur J Epidemiol. 2013;28(1):1-19.

19. Backes CH, Markham K, Moorehead P, Cordero L, Nankervis CA, Giannone PJ. Maternal Preeclampsia and Neonatal Outcomes. Journal of Pregnancy. 2011;2011:1-7.

20. Husiev VM, Khapchenkova DS. Influence of the transferred syphilitic infection on the course of pregnancy, childbirth and the state of newborns. Wiad Lek. 2019;72(2):175-80.

21. Hook EW. Syphilis. The Lancet. 2017;389(10078):1550-7.

22. Soares KKS, Prado TND, Zandonade E, Moreira-Silva SF, Miranda AE. Spatial analysis of syphilis in pregnancy and congenital syphilis in the state of Espirito Santo, Brazil, 2001-2018. Epidemiol Serv Saude. 2020;29(1):e2018193.

23. Hong FC, Liu JB, Feng TJ, Liu XL, Pan P, Zhou H, et al. Congenital syphilis: an economic evaluation of a prevention program in China. Sex Transm Dis. 2010;37(1):26-31.

24. Machado ES, Krauss MR, Megazzini K, Coutinho CM, Kreitchmann R, Melo VH, et al. Hypertension, preeclampsia and eclampsia among HIV-infected pregnant women from Latin America and Caribbean countries. J Infect. 2014;68(6):572-80.

25. Qin JB, Feng TJ, Yang TB, Hong FC, Lan LN, Zhang CL, et al. Synthesized prevention and control of one decade for mother-to-child transmission of syphilis and determinants associated with congenital syphilis and adverse pregnancy outcomes in Shenzhen, South China. Eur J Clin Microbiol Infect Dis. 2014;33(12):2183-98.

26. Dou L, Wang X, Wang F, Wang Q, Qiao Y, Su M, et al. Epidemic Profile of Maternal Syphilis in China in 2013. Biomed Res Int. 2016;2016:9194805.

27. Soeiro CM, Miranda AE, Saraceni V, Santos MC, Talhari S, Ferreira LC. Syphilis in pregnancy and congenital syphilis in Amazonas State, Brazil: an evaluation using database linkage. Cad Saude Publica. 2014;30(4):715-23.

28. Muricy CL, Pinto Junior VL. Congenital and maternal syphilis in the capital of Brazil. Rev Soc Bras Med Trop. 2015;48(2):216-9.

29. Tareke K, Munshea A, Nibret E. Seroprevalence of syphilis and its risk factors among pregnant women attending antenatal care at Felege Hiwot Referral Hospital, Bahir Dar, northwest Ethiopia: a cross-sectional study. BMC Research Notes. 2019;12(1).

30. Zhou H, Chen XS, Hong FC, Pan P, Yang F, Cai YM, et al. Risk factors for syphilis infection among pregnant women: results of a case-control study in Shenzhen, China. Sexually Transmitted Infections. 2007;83(6):476.

31. Woznicova V, Smajs D, Wechsler D, Matejkova P, Flasarova M. Detection of Treponema pallidum subsp. pallidum from skin lesions, serum, and cerebrospinal fluid in an infant with congenital syphilis after clindamycin treatment of the mother during pregnancy. $\mathrm{J}$ Clin Microbiol. 2007;45(2):659-61.

32. Wendel GD Jr, Sanchez PJ, Peters MT, Harstad TW, Potter LL, Norgard MV. Identification of Treponema pallidum in amniotic fluid and fetal blood from pregnancies complicated by congenital 
syphilis. Obstet Gynecol. 1991;78(5 Pt 2):890-5.

33. FG H, MA M, AJ S. C-reactive protein and cardiovascular disease: Lessons learned from studying genetically engineered mice. Nagasawa S ed C-Reactive Protein - N Res. 2008.

34. Zhu A, Wang C, Sun H, Han H, Wang F, Zhang L, et al. Circulating levels of Th1- and Th2-chemokines increase in patients with early syphilis. Chinese Journal of Cellular Molecular Immunology. 2017;33(3):380.

35. Novikov lu A, Novikov Al, Repina TV, Radul EV, Romanov AA. Changes in markers of systemic inflammatory response and functional state of the vascular wall in patients with early syphilis. Vestn Ross Akad Med Nauk. 2010(5):21-4.

36. Agita A, Alsagaff MT. Inflammation, Immunity, and Hypertension. Acta Med Indones. 2017;49(2):158-65.

37. Androulakis ES, Tousoulis D, Papageorgiou N, Tsioufis C, Kallikazaros I. C. S. Essential hypertension: is there a role for inflammatory mechanisms? Cardiol Rev. 2009;17(5):216-21.

38. Hage FG. C-reactive protein and hypertension. J Hum Hypertens. 2014;28(7):410-5.

39. Liu Q, Zhang XXW,YK, Li JH, Wang L. Correlation between pregnancy-induced hypertension and age in pregnant women from Hebei province, 2016. Zhonghua Liu Xing Bing Xue Za Zhi. 2018;39(9):1270-3.

40. Fruscalzo A, Bertozzi S, Londero AP, Biasioli A, Driul L, Kiesel L, et al. Menstrual abnormalities and predisposition to pregnancy-related hypertensive disorders: a retrospective study. Gynecol Endocrinol. 2010;26(6):445-50.

41. Fenzl V, Flegar-Mestric Z, Perkov S, Andrisic L, Tatzber F, Zarkovic N, et al. Trace elements and oxidative stress in hypertensive disorders of pregnancy. Arch Gynecol Obstet. 2013;287(1):19-24.

42. Das SS, Dhall GI, Dhall K, Dash S. Significance of serum iron levels as a biochemical marker in pregnancy-induced hypertension. International Journal of Gynaecology Obstetrics the Official Organ of the International Federation of Gynaecology Obstetrics. 1994;45(1):3.

43. Lewandowska M, Sajdak S, Lubinski J. Can Serum Iron Concentrations in Early Healthy Pregnancy Be Risk Marker of Pregnancy-Induced Hypertension? Nutrients. 2019;11(5).

44. Tande DL, Ralph JL, Johnson LK, Scheett AJ, Hoverson BS, Anderson CM. First trimester dietary intake, biochemical measures, and subsequent gestational hypertension among nulliparous women. J Midwifery Womens Health. 2013;58(4):423-30.

45. Zeng Y, Li M, Chen Y, Wang S. Homocysteine. endothelin-1 and nitric oxide in patients with hypertensive disorders complicating pregnancy. Int J Clin Exp Pathol. 2015;8(11):15275-9.

46. Zhao CH, Xu FS, Hu HY. A study on the correlation between blood lipids, uric acid and the progression of hypertension in pregnancy. Digest of world latest medical information. 2018;18(34):38-9.

47. Shen H, Liu X, Chen Y, He B, Cheng W. Associations of lipid levels during gestation with hypertensive disorders of pregnancy and gestational diabetes mellitus: a prospective longitudinal cohort study. BMJ Open. 2016;6(12):e013509. 
Figures

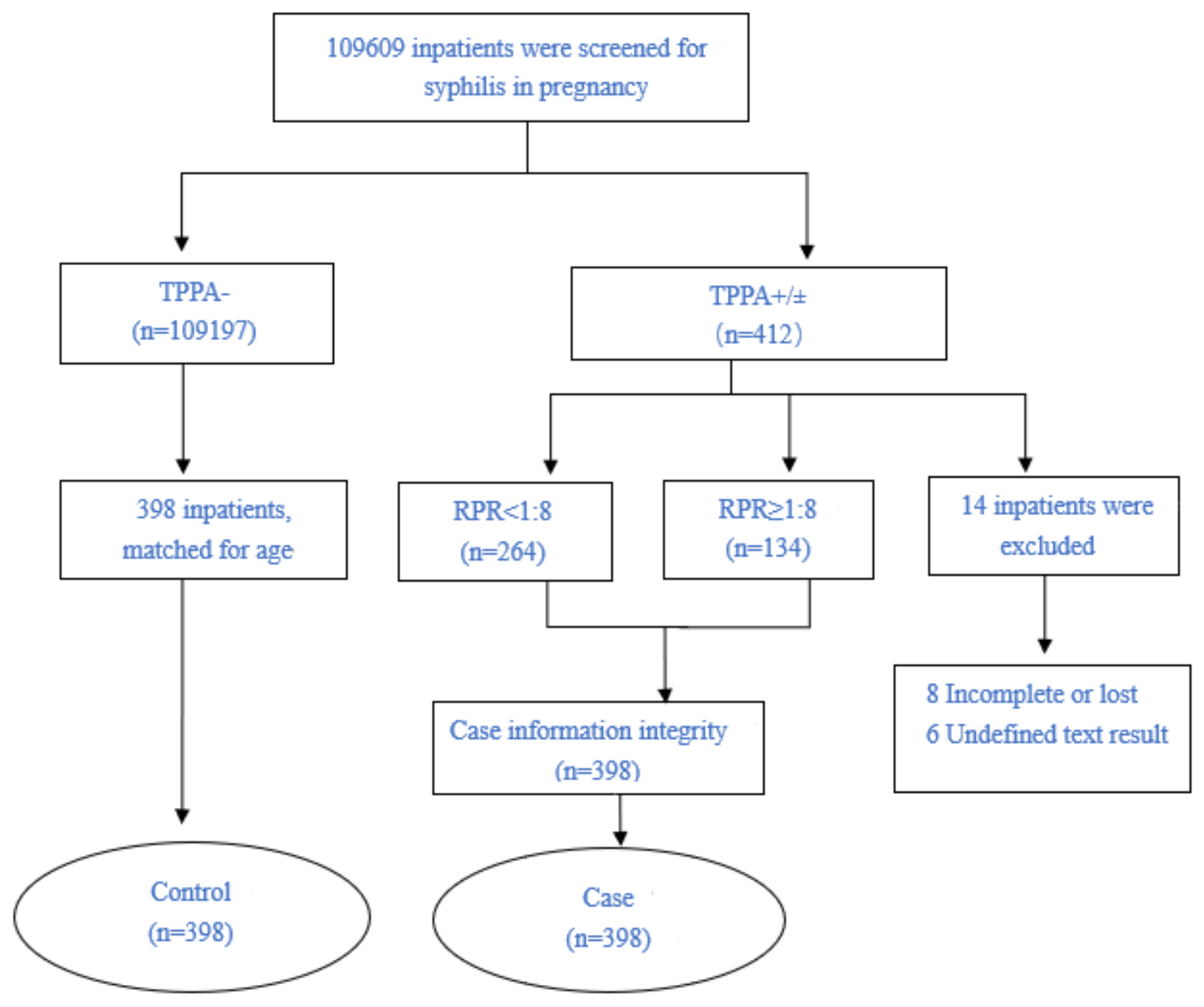

Figure 1

Selection criteria of the study subjects. 